\title{
DESCRIPTION DU TRÉMATODE STRIGEA GEODUBOISI n. sp. PARASITE D'UN CICONIIFORME AFRICAIN
}

\author{
Par Alain G. CHABAUD, Yves J. GOLVAN et René ROUSSELOT
}

L'autopsie d'un Ciconiiforme : Dissoura episcopus microscelis, mort au jardin zoologique de Brazzaville (Afrique équatoriale française), a permis de récolter dans l'intestin une dizaine de Strigéides remarquables par la structure de la région céphalique. L'espèce paraît nouvelle et nous en donnons ici la description.

\section{Description (1)}

Corps allongé, fortement courbé sur son bord dorsal, formé d'un segment antérieur, détaché du segment postérieur par une profonde incisure dorsale. Les téguments de la région céphalique sont ornés de petites épines lancéolées, larges de 2 à $3 \mu$ et pouvant atteindre une longueur de $10 \mu$. Ces épines sont très denses en avant et se raréfient dans le $1 / 3$ postérieur du segment antérieur. Muscle longitudinal parenchymateux très fort, étendu tout le long du bord dorsal jusqu'à la bourse copulatrice, avec de fortes expansions ventrales dans la portion antérieure du segment postérieur.

Segment antérieur subglobuleux, le diamètre ventro-dorsal un peu plus faible que le diamètre transversal et que le diamètre antéro-postérieur. Ventouse orale petite, portée par un petite lobe charnu, entourée d'une paire d'auricules généralement saillantes au-dessus du corps. Chaque auricule est formée d'un feuillet dorsal haut de $300 \mu$ et large de $500 \mu$ et d'un feuillet ventral de même hauteur qui vient fusionner avec celui du côté opposé devant la face ventrale de la ventouse. La ligne de fusion des deux feuillets récurrents ménage un espace libre devant la ventouse. Donc, la ventouse orale est située dans une véritable cupule ouverte en avant et légèrement encochée sur la ligne médio-ventrale. La ven-

(1) La nomenclature employée est celle de Dubois (1938).

Ann. de Parasitologie, T. XXXI, N ${ }^{\circ}$ 5-6. -1956. 


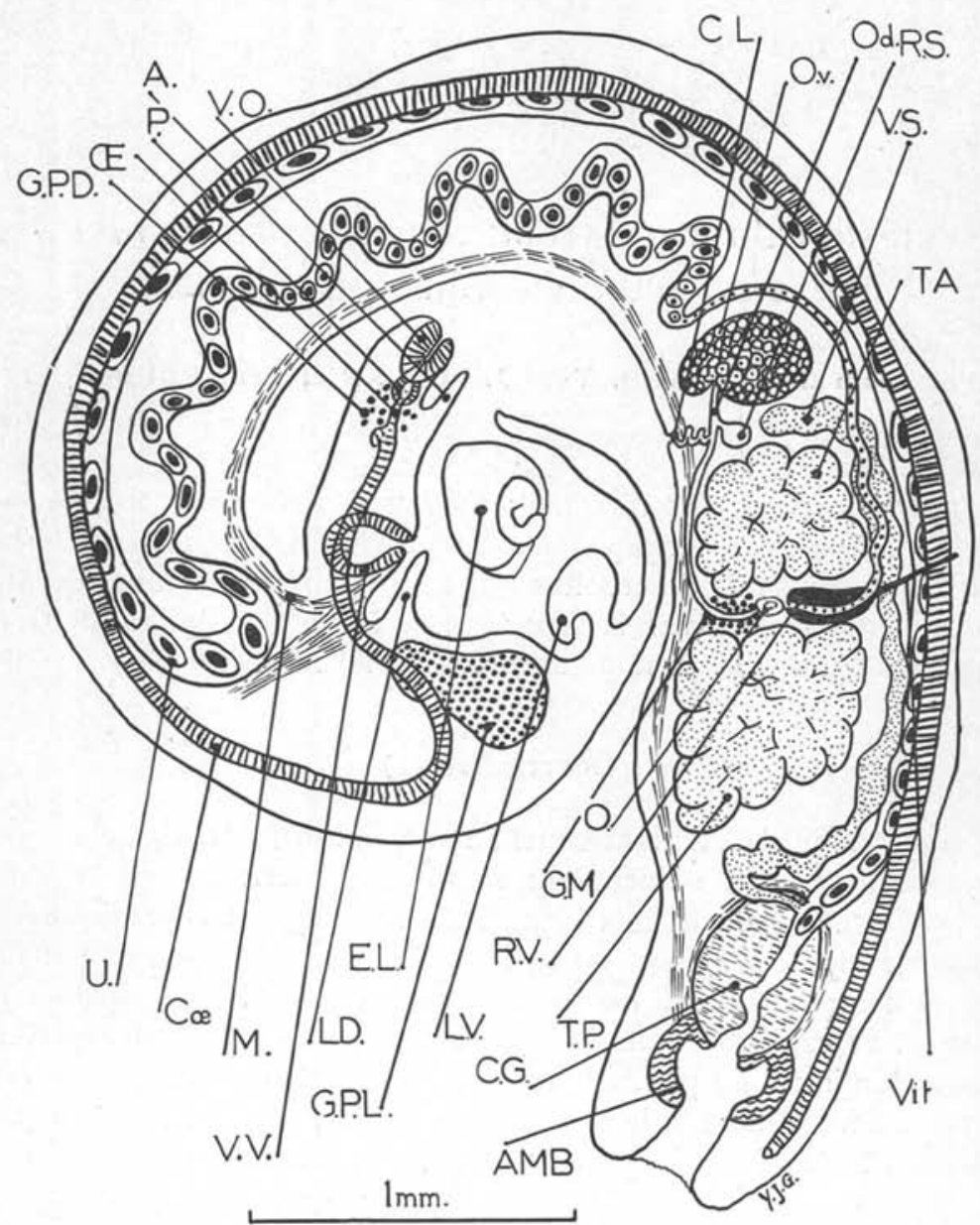

FIg. 1. - Schéma synthétique de l'organisation générale reconstituée d'après l'étude des coupes sériées. Signification des lettres de rappel indiquée dans la légende de la fig. 3 .

touse ventrale est nettement plus grande que l'orale. Elle occupe presque toute l'épaisseur de la paroi dorsale du segment antérieur.

L'organe tribocytique a une structure complexe et il est nécessaire de pratiquer des dissections et des coupes sériées pour pouvoir retrouver la disposition fondamentale du genre (un lobe ventral et un lobe dorsal). Le lobe ventral est représenté par un véritable bourrelet circulaire naissant ventralement à l'acétabulum et formant un cercle qui occupe le fond de la cavité du segment 
A

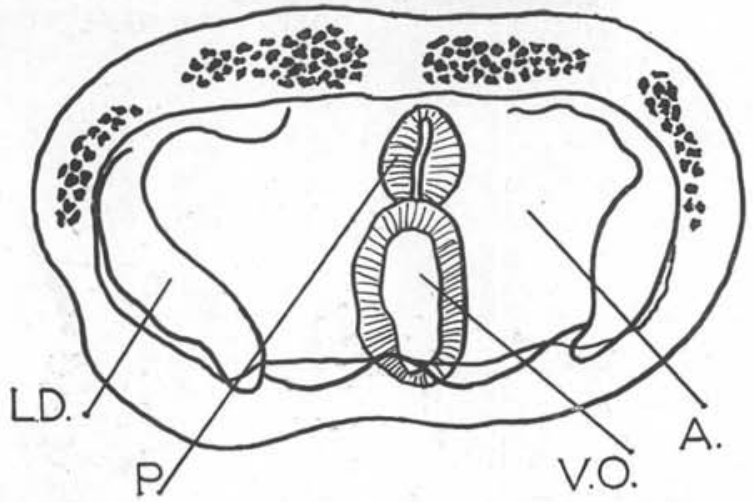

B

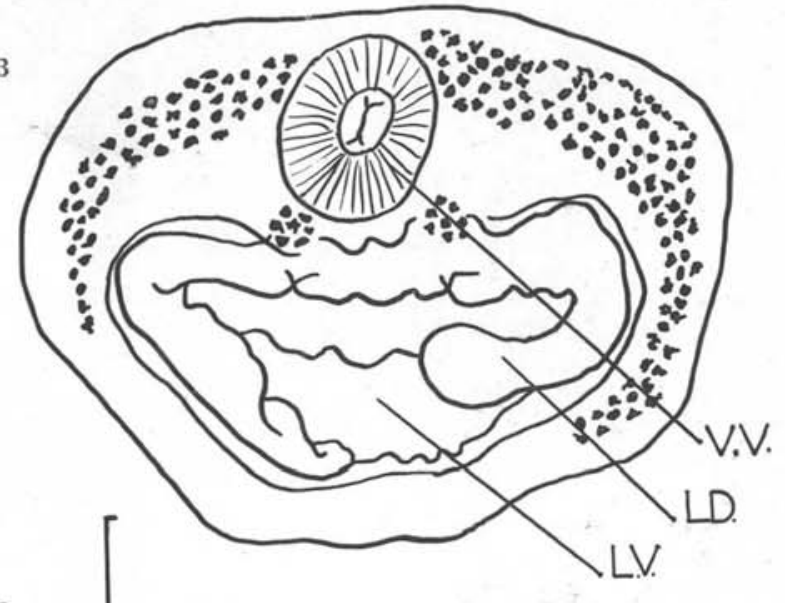

C

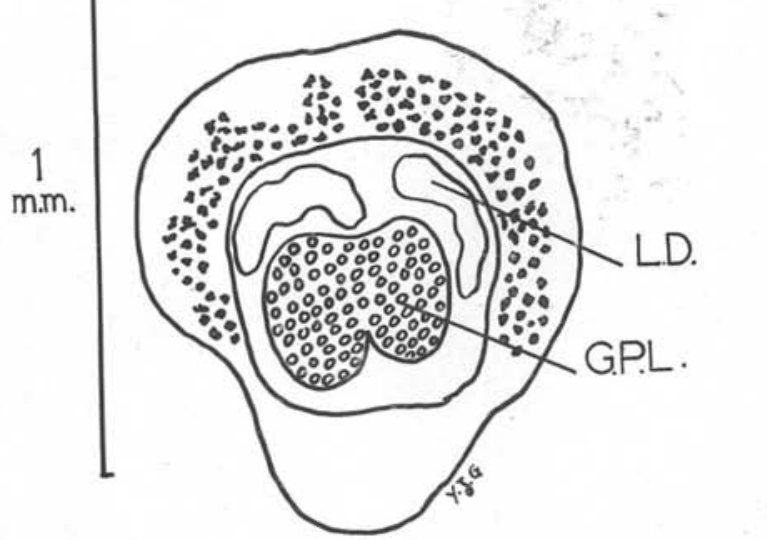

Fig. 2. - A : Vue apicale de l'extrémité antérieure. B : Coupe transversale passant au niveau de l'acétabulum. C : Coupe transversale passant au niveau de la glande protéolytique. Signification des lettres de rappel indiquée dans la légende de la fig. 3.

Ann. de Parasitologie, $\mathrm{T}$. XXXI, $\mathrm{N}^{\circ} 5-6 .-1956$. 


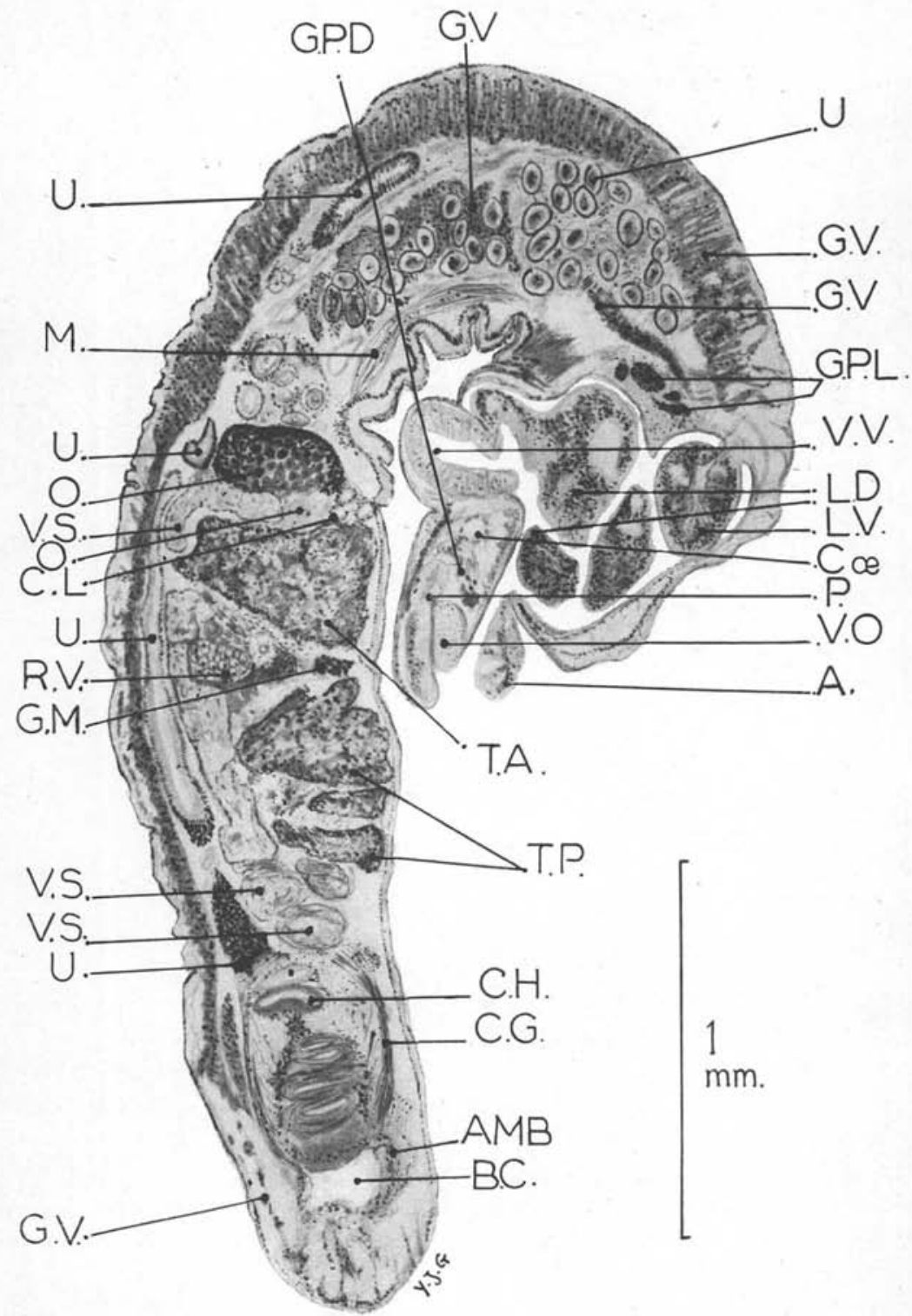

Fıg. 3. - Coupe sagittale du corps. Coloration Hématéine-éosine. A : Auricule. AMB : Anneau musculaire de la bourse copulatrice. BC : Bourse copulatrice ('extrémité postérieure n'est pas parfaitement orientée car la coupe n'est plus sagittale). CG : Cône génital. CH : Canal hermaphrodite. CL : Canal de Laurer. Cæ : Cæcum digestif. EL : Expansion latérale du lobe dorsal. GM: Glande de Mehlis. GPD : Glandes prosdétiques. GPL : Glandes pro- 
antérieur. Sur coupe, on distingue un feuillet périphérique fixé à la paroi et un feuillet interne dont le bord reste libre. Le lobe dorsal est représenté par deux feuillets indépendants qui naissent eux aussi devant l'acétabulum, puis s'élèvent au-dessus du lobe ventral en décrivant chacun un tour de spire; ils occupent ainsi à eux seuls toute la partie supérieure de la cavité du segment antérieur. Glande protéolytique assez large dans ses diamètres tranversal et dorso-ventral, mais réduite dans l'axe antéro-postérieur. Pharynx plus petit que la ventouse orale, mais bien marqué. Glandes prosdétiques abondantes, colorées en rouge vif par l'éosine. Esophage
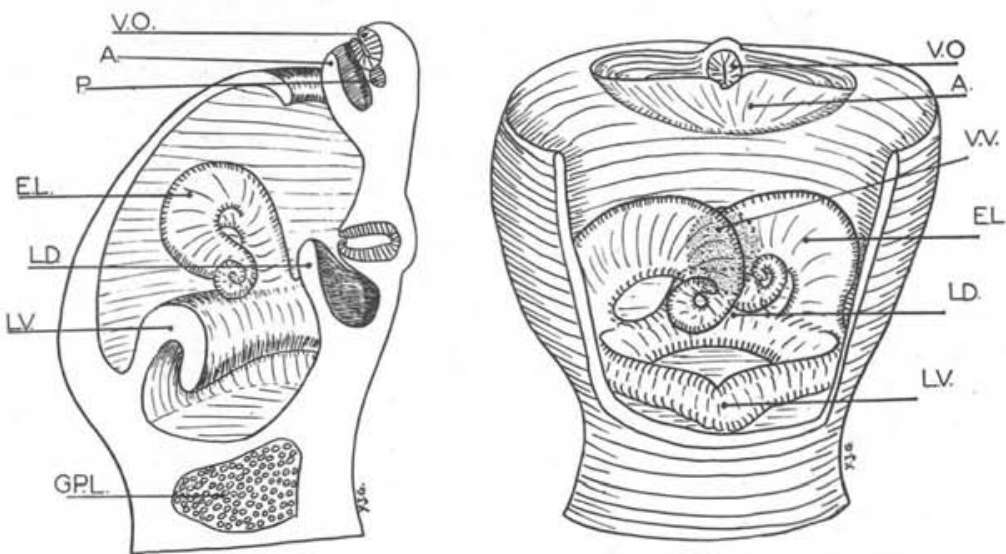

F1G. 4. - Schéma de la constitution de l'organe tribocytique. A : Coupe parasagittale. B : Vue ventrale après résection de la paroi ventrale du segment antérieur.

très court. Les deux branches intestinales passent de chaque côté de la glande protéolytique et gagnent le bord ventral du corps. Elles descendent entre les nappes vitellogènes ventrales et l'utérus dorsal jusqu'au niveau de la bourse copulatrice.

Ovaire subsphérique, beaucoup plus petit que les testicules, situé à la partie moyenne du segment postérieur. Peu après sa naissance, l'oviducte reçoit sur son côté dorsal le canal de Laurer

téolytiques. GV : Follicules vitellogènes. LD : Lobe dorsal de l'organe tribocytique. LV : Lobe ventral de l'organe tribocytique. M. Muscle parenchymateux longitudinal. Od : Oviducte. Oe : Esophage. Oo : Ootype. Ov : Ovaire. P : Pharynx. RV : Réservoir vitellin. RS : Réceptacle séminal. TA : Testicule antérieur. TP: Testicule postérieur. U : Utérus. Vit : Vitelloducte. VO: Ventouse orale. VV: Ventouse ventrale. VS : Vésicule séminale. 
et sur son côté ventral le canal du réceptacle séminal. Le réceptacle séminal est situé contre la partie antérieure de la vésicule séminale et, bien qu'il semble n'y avoir aucune connexion entre les deux organes, ils sont difficiles à distinguer l'un de l'autre. Le réceptacle séminal a cependant une paroi plus épaisse. Le premier

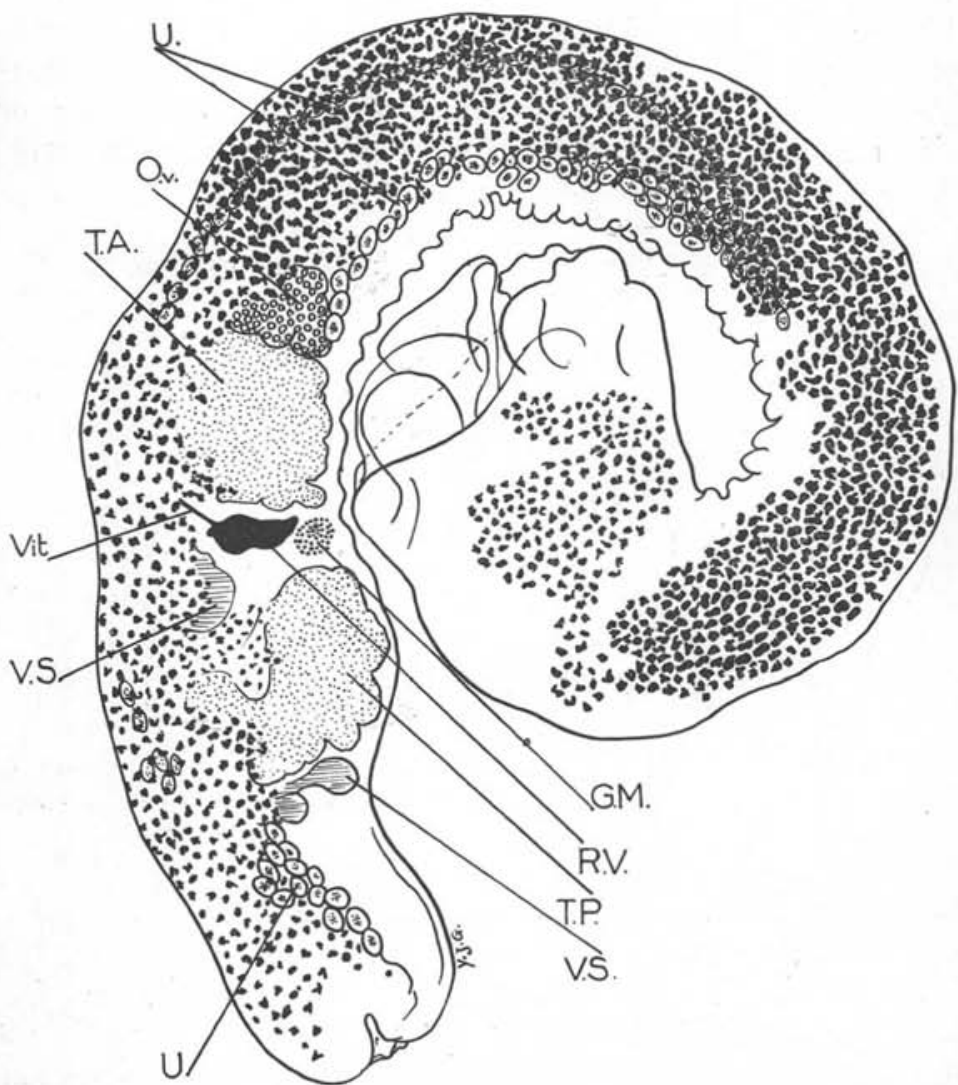

Fig. 5. - Vue latérale d'un spécimen éclairci au lacto-phénol.

Le lobe dorsal de l'organe tribocytique est évaginé en avant du segment antérieur

carrefour génital est très riche en spermatozoïdes et le canal de Laurer en est plein. Comme on voit au contraire très peu de spermatozoïdes dans le reste du tractus génital femelle, on peut supposer qu'il existe, dans le cas de cette espèce, une fécondation s'effectuant par le canal de Laurer. L'oviducte, après avoir reçu le canal de Laurer et le réceptacle séminal, se dirige postérieurement et contourne le testicule antérieur sur son bord dorsal. Il traverse 
alors une volumineuse glande de Mehlis, située dans la partie dorsale de l'espace intertesticulaire, puis entre en contact avec le réservoir vitellin qui occupe la partie ventrale de l'espace intertesticulaire. L'utérus contourne alors le bord ventral du testicule antérieur, puis décrit de nombreuses sinuosités ascendantes dans la partie antérieure du segment postérieur. Il se poursuit par une branche descendante qui longe le bord ventral du ver, dorsalement aux vitellogènes, et s'abouche enfin au sommet du cône génital. Follicules vitellogènes formant dans le segment antérieur deux lames latéro-ventrales à densité relativement faible, bien qu'ils envahissent la base des lobes de l'organe tribocytique. Dans le segment postérieur, les follicules se développent en une masse très dense et très étendue jusqu'au niveau de l'ovaire, puis se réduisent et n'occupent plus que la moitié ventrale du corps où ils forment encore un ruban dense, envahissant très largement les parois de la bourse copulatrice. CEufs nombreux dans l'utérus (environ 200).

Les deux testicules occupent presque complètement le troisième quart du segment postérieur. Ils sont de taille subégale et sont multilobés. Les canaux déférents n'ont pas été repérés. La vésicule séminale commence en arrière de l'ovaire, au contact du réceptacle séminal, puis descend sur le bord ventral des testicules, ventralement par rapport à la branche descendante de l'utérus. Après quelques grosses sinuosités en arrière du testicule postérieur, la vésicule séminale s'abouche à la partie postérieure et dorsale du cône génital.

Cône génital très nettement délimité du parenchyme ; sa lumière est tapissée d'une curieuse paroi, très finement et très régulièrement granuleuse. Bourse copulatrice à pore terminal, reliée au cône génital par un très puissant anneau musculaire.

Nous donnons ici les principales dimensions de deux spécimens, l'un de petite taille étudié en coupes sériées, l'autre de grande taille étudié après éclaircissement dans le lacto-phénol. Le mode de préparation accentue la différence de taille et les divergences entre les deux séries de mensurations sont donc très importantes.

TABleaU DES PRINCIPALES DIMENSIONS

Longueur : $5,7 \mathrm{~mm} .-9,5 \mathrm{~mm}$.

Segm. ant. . . . . . . .

Segm. post. . . . . . . . .

Segm. post./segm. ant. = long./larg. segm. post. . .

\section{Longueur}

1,05-1,2 mm.

4,65-8,3

4,43-6,91

6,54-10,0

\section{Diamètre}

0,9-1,2 mm.

$0,730-0,830$ 
Diamètres :

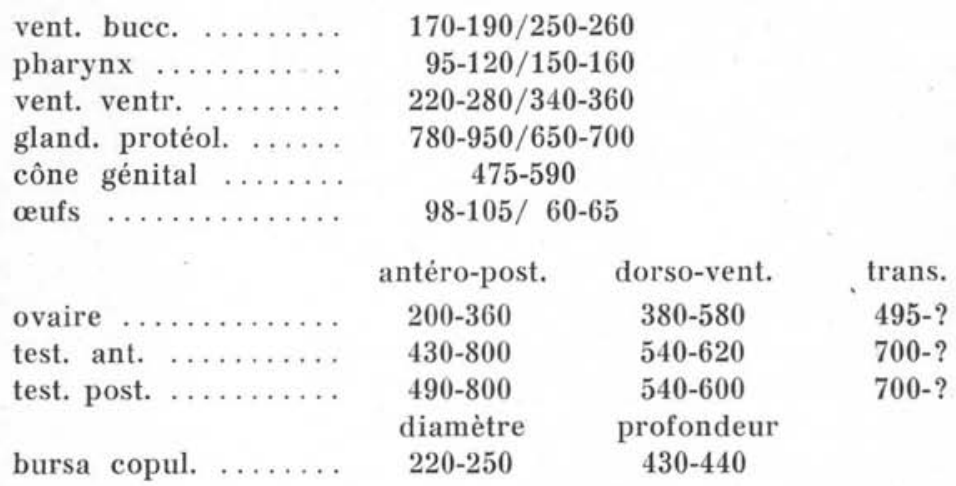

Distance : limit. $\beta$ vitlg. - extr. post. 210-220.

Situation dans segm. post.

$\gamma$ ovaire $\ldots \ldots \ldots \ldots$

$\alpha$ test. ant. .........

$51-56 / 100$

$\beta$ test. post. . . . . . .

$75-78 / 100$

limit. vitlg. . ........

$95-97 / 100$

\section{Discussion}

L'anatomie s'accorde à la définition générique du genre Strigea Abildgaard 1790, bien que la constitution de l'organe tribocytique soit plus complexe que ce qui est habituellement décrit pour les espèces du genre. On peut cependant résoudre cette structure en une disposition fondamentale en deux lobes : lobe ventral devenu un anneau circulaire occupant le fond de la cavité de l'organe tribocytique et lobe dorsal scindé en deux feuillets à disposition spirale doublant les parois latérales et antérieures de la cavité. L'anatomie génitale correspond tout à fait à celle de l'espèce type S. strigis (Schrank 1788), telle qu'elle est schématisée par Szidat (1928) (fig. 1). La seule différence est que nous individualisons un réceptacle séminal distinct de la vésicule séminale. La diagnose spécifique est rendue facile grâce à la monographie de Dubois (1938), que l'auteur a récemment mise à jour (1953). Depuis cette date, il faut ajouter, dans le genre, le $S$. eroliæ Fischer et Webster (1954), parasite d'un Charadriiforme nord-américain. Bien qu'elle ait un pharynx nettement marqué, notre espèce a quelques affinités avec certains Apharyngostrigea, parasites de Ciconiiformes. A. cornu (Zeder 1800) et A. egretii Verma 1936 sont beaucoup plus trapus. A. ramai (Verma 1936) aurait peut-être quelques affinités dans la structure du segment antérieur, mais les dimensions sont 
très différentes. Parmi les Strigea sensu stricto, l'espèce la plus proche semble être $S$. elongata Yamaguti 1935, parasite de Rapace au Japon. Les dimensions sont assez comparables, mais ni Yamaguti, ni Dubois qui a revu les spécimens types, n'ont constaté une structure particulière de l'organe tribocytique. D'autre part, les vitellogènes ne s'étendent pas dans la bourse copulatrice.

\section{RÉSUMÉ}

Description de Strigea geoduboisi n. sp., parasite dans l'intestin d'un Ciconiiforme, Dissoura episcopus microscelis, originaire du Moyen-Congo et mort au jardin zoologique de Brazzaville. L'espèce a tous les caractères correspondant au genre Strigea, mais l'organe tribocytique a subi une curieuse modification: le lobe ventral s'est transformé en un anneau postérieur et le lobe dorsal en deux lames spiralées antérieures.

D'autres caractères (existence d'un réceptacle séminal, distinct de la vésicule, présence de petites épines sur les téguments du segment antérieur) sont signalés, bien qu'ils ne soient peut-être pas caractéristiques de l'espèce.

\section{BibLIOGRAPHIE (1)}

Dubois (G.), 1938. - Monographie des Strigeida (Trematoda). Mém. Soc. Neuch. Sci. nat., VI, 535 pp., fig. 1-340, A-N.

- 1953. - Systématique des Strigeida (Complément de la monographie). Mém. Soc. Neuch. Sci, nat., VIII, $141 \mathrm{pp}$.

Fisher Jr. (F.) et Webster (J. Dan), 1954. - A new Strigeid from the pectoral sandpiper. J. Parasit., XL, 444-445, fig. 1-2.

Szidat (L.), 1928. - Beiträge zur Kenntnis der Gattung Strigea (Abildg.). Allgemeiner Teil : Untersuchungen über die Morphologie, Physiologie und Entwicklungsgeschichte der Holostomiden nebst Bemerkungen über die Metamorphose der Trematoden und die Phylogenie derselben. Zeitsch. f. Parasit., I, 612-687, 35 fig. + pl. 8 .

\section{Institut de Parasitologie, Faculté de Médecine de Paris et Service de l'élevage de Brazzaville}

(1) Note infrapaginale. - Depuis le dépôt du texte de ce travail, plusieurs Strigeides africains ont été décrits : trois espèces nouvelles parasites de Crocodiles et quatre parasites de Rapaces. Aucune de ces espèces ne possède des caractères anatomiques assez proches de la nôtre pour que nous ayons besoin de discuter la validité de $S$. geoduboisi n. sp. par rapport à elles.

Bisseru (B.), 1956. - On four new Trematodes of the Genus Strigea from Central African Birds of Prey. Jl. Helminthol., XXX (1), 63-79, fig. 1-11.

1956. - On three new Species of Strigeid Trematodes from an African Crocodile and the erection of a new Family, Neostrigeida. Jl. Helminthol., XXX (4), 217-232, fig. 1-15. 